\title{
Accelerating the development of innovative cellular therapy products for the treatment of cancer
}

\author{
Mark D. Stewart ${ }^{1, *}$, Anne Keane ${ }^{2}$, Lisa H. Butterfield ${ }^{3}$, Bruce L. Levine ${ }^{4}$, Bruce Thompson ${ }^{2}$, \\ Yuan $\mathrm{Xu}^{5}$, Chris Ramsborg ${ }^{6}$, Ann Lee ${ }^{6}$, Michael Kalos ${ }^{7}$, Chin Koerner ${ }^{8}$, Timothy Moore ${ }^{9}$, \\ Ingrid Markovic ${ }^{10}$, Laura Lasiter $^{1}$, Ramy Ibrahim ${ }^{3}$, Jeffrey Bluestone ${ }^{3,11}$, Ellen Sigal ${ }^{1}$, Jeff Allen ${ }^{1}$ \\ ${ }^{1}$ Friends of Cancer Research, Washington, DC, USA \\ ${ }^{2}$ Lyell Immunopharma, San Francisco, California, USA \\ ${ }^{3}$ Parker Institute for Cancer Immunotherapy, San Francisco, California, USA \\ ${ }^{4}$ University of Pennsylvania, Philadelphia, Pennsylvania, USA \\ ${ }^{5}$ Legend Biotech, Piscataway, New Jersey, USA \\ ${ }^{6}$ Juno Therapeutics, A Bristol-Myers Squibb Company, Seattle, Washington \\ ${ }^{7}$ Arsenal Biosciences, South San Francisco, California, USA \\ ${ }^{8}$ Novartis Pharmaceuticals, Rockville, Maryland, USA \\ ${ }^{9}$ PACT Pharma, South San Francisco, California, USA \\ ${ }^{10}$ Genentech, A Member of the Roche Group, Washington, DC, USA \\ ${ }^{11}$ University of California San Francisco, San Francisco, California, USA
}

\section{A R T I C L E I N F O}

\section{Article History:}

Received 5 December 2019

Accepted 27 January 2020

\section{Key Words:}

\section{cell therapy}

clinical development

manufacturing

regulatory policy

t-cell-based therapies

\begin{abstract}
A B S T R A C T
The field of cell therapy is rapidly emerging as a priority area for oncology research and drug development. Currently, two chimeric antigen receptor T-cell therapies are approved by the US Food and Drug Administration and other agencies worldwide for two types of hematologic cancers. To facilitate the development of these therapies for patients with life-threatening cancers with limited or no therapeutic options, scienceand risk-based approaches will be critical to mitigating and balancing any potential risk associated with either early clinical research or more flexible manufacturing paradigms. Friends of Cancer Research and the Parker Institute for Cancer Immunotherapy convened an expert group of stakeholders to develop specific strategies and proposals for regulatory opportunities to accelerate the development of cell therapies as promising new therapeutics. This meeting took place in Washington, DC on May 17, 2019. As academia and industry expand research efforts and cellular product development pipelines, this report summarizes opportunities to accelerate entry into the clinic for exploratory studies and optimization of cell products through manufacturing improvements for these promising new therapies.
\end{abstract}

(c) 2020 International Society for Cell and Gene Therapy. Published by Elsevier Inc. This is an open access article under the CC BY-NC-ND license. (http://creativecommons.org/licenses/by-nc-nd/4.0/)

\section{Introduction}

Advancements in cancer immunology and recent clinical experience with emerging cellular therapeutics, such as tumor-infiltrating lymphocytes, engineered $\mathrm{T}$-cell receptor $\mathrm{T}$ cell and chimeric antigen receptor (CAR) T-cell therapies, are generating huge interest and activity both academically and industrially. Additional platforms and technologies, including cellular therapies based on natural killer and other immune cells, as well as novel gene-editing approaches have entered into clinical trials. These emerging therapeutics have the

\footnotetext{
* Correspondence: Mark Stewart, PhD, Friends of Cancer Research, 1800 M Street NW, Washington, DC 20036, USA.

E-mail address: mstewart@focr.org (M.D. Stewart).
}

potential to rapidly change cancer treatment and represent a new paradigm in medical therapy for cancer.

To date, CAR T-cell therapies have been approved by the US Food and Drug Administration (FDA) only for certain types of leukemia and lymphoma; other T-cell based therapies have shown remarkable activity in a limited number of solid tumors but have not yet progressed to approval [1-5]. There is great interest in exploring these new treatment modalities to encompass the treatment of solid tumors, which comprise $90 \%$ of all cancers and the majority of cancer deaths [6]. Currently, multiple scientific challenges exist for the successful use of T-cell-based therapies in solid tumors, including issues related to target antigen selectivity and expression, the immunosuppressive nature of the tumor microenvironment, tumor T-cell infiltration and T-cell exhaustion. Academia and industry are attempting to address these barriers, and numerous T-cell-based product 
candidates are being developed, involving various cell subtypes, autologous and allogeneic approaches, molecular engineering strategies and many different antigen targets. However, pre-clinical in vivo animal models are limited in their ability to predict product safety and efficacy for T-cell-based therapeutics, which limits progress.

To advance these therapies ultimately for a larger number of patients, and in particular for those with solid tumors, it would be desirable to design small, data-intensive exploratory clinical studies to differentiate which approaches warrant continuation and further focus. These studies would provide an opportunity to optimize the candidates to subsequently advance into full product development by generating knowledge that cannot be gained using currently available pre-clinical models. Small, early clinical studies also have the potential to facilitate a better understanding of the biology of T-cell-based therapeutics and the product attributes driving efficacy and safety. However, clinical data can be obtained only after the compilation and submission of an investigational new drug application (IND) for each candidate to be evaluated. These IND procedural requirements can make it prohibitively slow and expensive to pursue this critical opportunity for more than a select few product candidates. This can be particularly problematic for academic researchers and small biotechnology companies who generate much of the most innovative science in the field.

Furthermore, there can be varying interpretations of FDA guidance regarding phase appropriate current Good Manufacturing Practice (cGMP) requirements for reagents, plasmids, peptides, vectors and T-cell infusion products for use in the early investigational setting. Consequently, some institutions have imposed very strict cGMP requirements on all investigators that are more applicable for later-stage clinical development. These strict requirements significantly increase the cost and time for academic centers and industry to manufacture early investigational cell products and extend the time to evaluate which approaches should be taken to late-stage/ pivotal clinical trials.

Ensuring that the most effective T-cell-based therapeutics are developed for the largest number of patients requires the adoption of a new adaptive manufacturing paradigm as more patients are treated, and more clinical, translational and product quality data are collected during a product lifecycle. In the late-stage development and post-licensure settings, as product and process knowledge increases, a strategy that enables adjustment of the manufacturing process conditions based on patient or patient-specific raw material information to maximize product quality for all patients would be beneficial without the need to conduct costly and lengthy studies. Furthermore, there is an opportunity to develop a regulatory framework for expedited clinical development to facilitate this adaptive learning-based manufacturing paradigm to allow for patient-level modifications for a subset cohort of patients, especially as understanding of the linkage between product quality attributes, manufacturing processes, clinical efficacy and safety evolves through late-stage development and post licensure.

\section{Opportunities to Accelerate Early Discovery Through IND Application Flexibility}

The FDA's 2006 Exploratory IND Studies Guidance acknowledged the need to reduce the time and resources expended on candidate products that are unlikely to proceed to licensure and described early phase 1 exploratory approaches that are consistent with regulatory requirements and maintain needed human subject protection, but which involved lesser requirements and lower costs, enabling sponsors to progress more efficiently [7]. This guidance also acknowledged that there is a great deal of flexibility in the amount of data that needs to be submitted with an IND application.

Application of the exploratory IND concept to early, small clinical studies for the purpose of candidate selection for T-cell-based therapeutics would facilitate the critical opportunities described above. However, modifications would be needed. The current guidance explicitly states that an exploratory IND study is intended to involve very limited human exposure and to have no therapeutic or diagnostic intent. Post-infusion expansion of cellular therapies, the durable nature of cellular products and the ethical requirement to ensure clinical equipoise for patients with life-threatening cancers necessitate that they be dosed at therapeutic levels and with therapeutic intent. Nonetheless, a science- and risk-based approach to an expansion of the exploratory IND concept as it is applied to T-cell-based therapies is possible and appropriate. This would facilitate the evaluation of the safety and activity of next-generation T-cell-based therapeutics that could fundamentally improve their efficacy via small, data-intensive clinical studies.

An expanded exploratory IND pathway would facilitate the efficient generation of clinical data on multiple T-cell-based product candidates or hypotheses in small ( $\mathrm{N}$ generally less than 30 patients per cohort) studies, reducing the regulatory burden for the sponsor. To ensure patient protection, enrollment in exploratory studies should be limited to patients with advanced cancers of unmet need and limited or no treatment alternatives. The total numbers of patients to be treated under an exploratory IND should be limited to the number required to evaluate the hypotheses to be tested.

Exploratory-phase protocols should be designed with a focus on patient safety and should incorporate opportunities to minimize risks. Therefore, appropriate consideration should be given to protocol design features (Table 1) [8,9].

The following sections outline how phase-appropriate cGMP compliance focused on product quality and patient safety and a streamlined parent-child IND alternative to the current single IND per drug product process would further facilitate the conduct of these studies under an expanded exploratory IND paradigm. T-cell-based therapies are used as specific examples, although the proposal could apply to other cellular products. Table 2 provides a summary of proposed strategies to facilitate the development of cellular therapies.

\section{Phase Appropriate cGMP Compliance Focused on Product Quality and Patient Safety}

The US FDA's 2008 Guidance for Industry: cGMP for Phase 1 Investigational Drugs provides a framework whereby more phase-appropriate manufacturing can occur for early studies [10]. The recognition that smaller-scale manufacturing processes may be excluded from some of the controls required for later stages of development where larger numbers of patients are exposed to treatment or for commercialization is critical to support innovative research and establish a better understanding of the biological impact of new therapeutics in small investigational human studies. However, consistent understanding and interpretation of this guidance, especially as it would apply to exploratory cellular therapy INDs, is needed. We provide several key examples below where explicit alignment between FDA, academic and government institutions and industry would facilitate the early exploratory clinical studies described above.

Table 1

Protocol design features to minimize risk.

Judicious dose escalation, cohorts and DLT windows

Adequate dosing intervals and safety assessments

Ongoing assessment by a safety monitoring committee

Incorporation of pre-specified safety, efficacy and futility decision points (Simon two-stage design)

Pre-planned early reporting of safety results

Explicit characterization of studies as "exploratory" in protocol and informed consent form

DLT, dose-limiting toxicity. 
Table 2

Summary of strategies to facilitate development of cellular therapies.

\begin{tabular}{|c|c|}
\hline Strategy & Description/process \\
\hline \multicolumn{2}{|l|}{ Early phase } \\
\hline Parent-child IND & $\begin{array}{l}\text { Parent IND would contain common } \\
\text { sections providing all relevant } \\
\text { information for the candidates or } \\
\text { manufacturing alterations. Each } \\
\text { child IND would cross-reference } \\
\text { common sections while providing } \\
\text { only the candidate- or process- } \\
\text { specific information. }\end{array}$ \\
\hline Phase-appropriate cGMP compliance & $\begin{array}{l}\text { Phase0appropriate manufacturing } \\
\text { requirements would focus on } \\
\text { product quality and patient safety } \\
\text { using a risk-based approach to } \\
\text { enable more efficient manufactur- } \\
\text { ing processes in early-phase } \\
\text { development. }\end{array}$ \\
\hline Exploratory IND paradigm & $\begin{array}{l}\text { Enrollment in trials with exploratory } \\
\text { cellular therapy INDs would be } \\
\text { limited to patients with advanced } \\
\text { cancers and limited or no treat- } \\
\text { ment alternatives. Early planned } \\
\text { safety reporting would support an } \\
\text { open regulatory dialogue. This } \\
\text { pathway would facilitate the effi- } \\
\text { cient generation of clinical data } \\
\text { and inform whether more formal } \\
\text { trials should be pursued. }\end{array}$ \\
\hline \multicolumn{2}{|l|}{ Late phase } \\
\hline Adaptive manufacturing process & $\begin{array}{l}\text { An adaptive manufacturing process } \\
\text { with the goal of generating a } \\
\text { highly similar drug product from } \\
\text { the patient-specific starting mate- } \\
\text { rial is needed. A regulatory strat- } \\
\text { egy that adjusts a process as } \\
\text { product and process knowledge } \\
\text { increases and based on patient or } \\
\text { patient-specific raw material } \\
\text { information to maximize product } \\
\text { quality for all patients will permit } \\
\text { the avoidance of extensive costly } \\
\text { and lengthy clinical studies. }\end{array}$ \\
\hline $\begin{array}{l}\text { Post-marketing product optimiza- } \\
\text { tion and modifications }\end{array}$ & $\begin{array}{l}\text { As we gain stronger product knowl- } \\
\text { edge and process understanding, } \\
\text { modifications to manufacturing } \\
\text { processes could be managed via a } \\
\text { pre-negotiated plan with health } \\
\text { authorities (e.g., Post-Approval } \\
\text { Lifecycle Management or Compa- } \\
\text { rability Protocol). Filing require- } \\
\text { ments for the change may include } \\
\text { a combination of an analytical } \\
\text { comparability assessment and/or a } \\
\text { small clinical study, analogous to a } \\
\text { bioequivalence study for a new } \\
\text { process. A post-market commit- } \\
\text { ment could be considered to dem- } \\
\text { onstrate/confirm the efficacy of } \\
\text { the new process. }\end{array}$ \\
\hline
\end{tabular}

Implicit in any approach for manufacturing phase 1-appropriate materials is a focus on patient safety, and the concepts below are proposed with an emphasis on risk assessments and analytical testing to determine and manage potential impact to patient safety. As such, Tcell-based cellular products would undergo release testing following manufacture for standard safety attributes, such as sterility, absence of mycoplasma and endotoxin, viral integration elements (vector copy number), identity, purity and potency.

We note that if remarkable efficacy were seen for a product development candidate tested in an exploratory IND, the requirement for a full IND with more burden of proof for manufacturing process and product knowledge and control would still apply with the potential for increased associated effort and cost. Sponsors may decide to mitigate this risk by pursuing limited process development activities in parallel with clinical studies under an exploratory IND.

A risk-based approach to requirements for the production of raw materials and drug substance (e.g., viral vectors) for T-cell-based therapeutics could more rapidly lead development teams to better test improved combinations of therapeutics, single-chain variable fragments alterations, novel manufacturing interventions, etc., which would lead to more robust and effective products that do not fail in later-stage development studies. Flexibility to permit the use of representative viral vectors not necessarily produced in a GMP facility, but rather in well-controlled laboratories, would result in significant monetary and time savings with little risk to patients. These opportunities could reduce the total time to manufacture investigational Tcell-based therapeutic candidates for use in an early clinical study under an exploratory IND by approximately $50 \%$, as depicted in Table 3 and described in greater detail in subsequent sections.

\section{Reduction in the infrastructure requirements for the manufacture of plasmids}

Currently, production of plasmid DNA for downstream production of viral vectors and/or for gene-editing tools is often outsourced to a limited number of companies, resulting in high costs and long manufacturing queues. Generally, sponsors and academic researchers have the technical capabilities to produce these plasmid DNA's, but interpretations of FDA guidance have led to local institutional policies requiring cGMP-grade plasmids for clinical studies. Due to the high infrastructure requirements (International Standards Organization-7 clean rooms, fully developed quality systems and cGMP trained personnel and associated resources) needed to produce cGMP-grade plasmid DNA, many institutions have not invested in the development of the manufacturing and quality infrastructure to produce these raw materials internally. In the industry setting, the impression that cGMP-grade plasmids may be required increases the cost and time associated with manufacturing investigational cellular products. Manufacture of cGMPgrade plasmids for small, exploratory clinical trials of multiple early cellular product candidates unnecessarily increases the cost and time to conduct these studies because it is expected that many of the candidates would not progress into full product development and licensure.

As an alternative to a requirement for cGMP-grade plasmids, highquality (HQ) fit-for-purpose plasmids may be acceptable. Plasmid DNA can be tested and characterized to confirm its suitability for downstream use in early, exploratory clinical trials with little risk to patient safety.

For example, the regulatory burden associated with the manufacture of HQ DNA plasmids for exploratory clinical studies could be reduced by eliminating the need for an Escherichia coli master cell bank. Note that a sponsor could also make a business decision to create the master cell bank and then freeze it, deferring the need for time-consuming and expensive testing until a decision was made to go forward with full development with that product candidate. Manufacturing could occur with review of production protocols, analytical results, manufacturing batch records and release tests performed by a second independent technical expert rather than quality assurance personnel. A certificate of testing (CoT) could be produced summarizing the test results. The authors proposed a representative fit-for-purpose CoT for plasmid DNA for early-phase clinical trials in a previous publication [11]. In essence, a CoT is similar to a certificate of analysis but differs in a few key elements: (i) tests are mostly compendial and may not be fully qualified/validated; (ii) tests may be peer reviewed by a technical expert (in lieu of a quality assurance resource); and (iii) test results have a Target Value in lieu of Acceptance Criterion. In addition, because the plasmid DNA materials are stable when frozen and anticipated to be used quickly in downstream manufacturing of viral vectors, at this stage the need to generate stability data could be weighed against the timing of use and available research data and, in some cases, waived. 
Table 3

Summary of efficiencies gained through early stage manufacturing.

\begin{tabular}{|c|c|c|c|c|}
\hline CMC activity & Typical time ${ }^{\mathrm{a}}$ investment & Areas of proposed flexibility & Potential time ${ }^{\mathrm{a}}$ savings & Potential cost savings ${ }^{\mathrm{c}}$ \\
\hline Use of $R \& D$ reagents & $3-6 \mathrm{mo}$ & $\begin{array}{l}\text { Increasing options for use of R\&D reagents and reduc- } \\
\text { ing cost and time to either enable or negotiate GMP } \\
\text { manufacture of reagents }\end{array}$ & $1-3 \mathrm{mo}$ & $\$-\$ \$ \$$ \\
\hline Plasmid manufacturing & $4 \mathrm{mo}(+3-6 \mathrm{mo}$ in queue) & $\begin{array}{l}\text { Reduced plasmid characterization and infrastructure } \\
\text { requirements }\end{array}$ & $5-7 \mathrm{mo}$ & $\$ \$$ \\
\hline Viral manufacturing & $6 \mathrm{mo}(+9-12 \mathrm{mo}$ in queue) & $\begin{array}{l}\text { Waive RCL testing in lieu of surrogate testing; } \\
\text { reduced cGMP requirements for ancillary reagents }\end{array}$ & $4 \mathrm{mo}$ & $\$$ \\
\hline Cell product engineering runs ( 3 runs) & $3 \mathrm{mo}$ & $\begin{array}{l}\text { Use representative pilot virus for parallel cell product } \\
\text { engineering runs }\end{array}$ & $2 \mathrm{mo}^{\mathrm{b}}$ & $\mathrm{N} / \mathrm{A}$ \\
\hline
\end{tabular}

Use of phase-appropriate vector testing strategies, including reductions in the replication competency testing requirements

The current replication competency virus assay is based on testing vector supernatant or end of production cells on susceptible human cells over an 8- to 10-week period; this requirement adds significant expense and time to vector release timelines, and, hence, to overall product manufacturing activities. Despite theoretical concerns, the risk of replication competency-related recombination events using third-generation viral vectors is extremely low because the elements required for virus replication are separated across three or four different plasmid DNAs and the 3' untranslated region portion of the transfer plasmids have been modified, resulting in transcriptional inactivation of the long terminal repeat in the proviruses after integration. With respect to viral vectors currently used in cell therapy products, researchers have documented that, to date, no viral vector recombination events have been observed in T cells across hundreds of patient product tests [12,13].

Alternative vector release testing based on a surrogate qualified/ validated qualitative polymerase chain reaction (qPCR) test for the glycosaminoglycans and vesicular stomatitis virus $G$ glycoprotein or similar envelope gene sequences depending on the viral vector pseudotype, as has been recently suggested by Skrdlant et al. may be acceptable, particularly in the context of early, exploratory studies in patients with limited or no remaining treatment options and poor long-term survival [14].

Vector and cellular drug product release decisions for such exploratory studies could be made on the basis of surrogate testing; if required, full, culture-based replication competency-based testing could be conducted in parallel in the background. The results of the full-culture testing would be available within the period of post-infusion patient follow-up during which time patients would be followed up for the development of treatment-related malignancy.

Use of a risk-based approach for determining safety of reagents used in early clinical trials

Extensive manufacturing requirements for reagents (e.g., activation beads, selection reagents, cytokines and recombinant growth factors) create a time and cost burden in early development. For early clinical trials, it may be reasonable to reduce these requirements, based on an appropriate risk assessment. Typically, these reagents are produced and stored frozen at higher concentrations to ensure greater stability. During manufacturing, a reagent is thawed and diluted to the working concentration and then added to a much larger culture volume. Unless the reagent is used constantly throughout the entire manufacturing process, several rounds of washing, media changes and formulation of the final cell product will significantly dilute the reagent. Similar to the manufacturing requirements for plasmid DNA, fit-for-purpose requirements (relying on science- and risk-based approaches to ensure patient safety and quality of the reagent) for HQ reagents used within the manufacturing process for early-phase clinical studies would significantly reduce the cost and time burden associated with using innovative reagents, while ensuring patient safety. An emphasis on risk assessments to identify potential impact to patients (e.g., sterility/bioburden and products of animal origin) could provide guidance to academic researchers and industry partners. For non-pharmacopoeial reagents of non-biological origin, a review of a CoT may provide assurance that a reagent is fit-for-purpose for use in the manufacturing of cellular products for small, early clinical studies. For reagents of biological origin (e.g., human serum), purchasing from an accredited supplier, along with a certificate of analysis (source, sterility, endotoxin, infectious agents, mycoplasma) can confer suitability of use. Academic or small industry sponsors may benefit particularly from opportunities to share costs and risks by collaborating on the development of peer-reviewed tests in lieu of certificates of analysis and the accreditation of suppliers. An illustrative example of the approach to characterization of a novel recombinant cytokine, based on the concepts provided in International Conference on Harmonisation of Technical Requirements for Registration of Pharmaceuticals for Human Use Q6B and other regulatory guidance, is available [11].

\section{Opportunities for flexibility in cell processing}

Given the resources required and complexity of manufacturing Tcell-based therapeutic products, identifying similar flexibilities in the cell-processing space would provide significant opportunities for innovation. Although a robust discussion of the kinds of flexibility desired is out of scope for this document, a few examples and the anticipated impacts are offered below. Typically, a T-cell-based therapeutic is engineered using a relatively similar set of manufacturing unit operations: acquisition of patient starting material through apheresis/leukapheresis, possible isolation/purification of the T cells through gradient, magnetic or alternative selection means, activation and retroviral transduction to introduce the CAR or T-cell receptor, expansion of the engineered $\mathrm{T}$ cells and final harvest and cryopreservation. Although there are variations on the above approach and a number of different pieces of equipment used in various manufacturing processes, the general process lends itself to some potential flexibilities in the early development space.

Flexibility to permit the use of representative viral vectors in cell product engineering runs

Current approaches to qualify a cellular therapy manufacturing process is often interpreted as requiring the use of GMP-grade viral vector in at least three engineering runs conducted to confirm the adequacy of the cellular product manufacturing process. Clarity that the use of the representative pilot (i.e., development-grade viral vectors 
manufactured in accordance with the final manufacturing process) would be acceptable could result in significant time savings because the cellular product engineering runs could be run in parallel with the actual GMP production runs for the viral vector. Additionally, because much of the development work for autologous cell therapies is done at scale, fewer engineering runs (e.g., two) would be reasonable. As such, data from both development runs (e.g., in the process development laboratory) and engineering runs (e.g., in the GMP manufacturing facility) could be combined to demonstrate adequate understanding and control of the process to support an IND submission.

\section{Phase-appropriate release testing}

For early-phase exploratory trials, a focus on testing cell product components related to safety can provide flexibility. Safety would be assessed via testing for sterility, mycoplasma (via a rapid testing paradigm), endotoxin, etc., which are each important to demonstrating a lack of contamination of the cell product. Testing the cell product for effectiveness of transduction through assessment of vector integration into the T-cell genome can be performed by determining the average vector copy number via qPCR. Additionally, surrogate measures of viral replication competency can be performed using qPCR with primers against elements of the viral genome (discussed above as part of replication competent retrovirus/replication competent lentivirus testing above). Identity, purity and potency are important release assays used to demonstrate that a particular manufacturing process was able to successfully yield the expected product. While identity and purity tests are generally required even in early phases, due to the complexity of cell products, potency assays may be in development or deferred in very early clinical phases in favor of a well-qualified dose-determining assay with incremental implementation of the potency assay as development proceeds.

Development of a parent-child IND framework to reduce the regulatory burden associated with the clinical testing of multiple potential product candidates

In the setting of small, exploratory early data-intensive clinical studies intended to investigate the safety, feasibility and mechanism of action of several closely related T-cell-based candidates or related manufacturing process alterations (e.g., process alterations to maintain stemness), a more efficient parent-child IND structure and process may be appropriate.

The parent IND submitted by a single sponsor/investigator would contain common sections providing all of the relevant information manufacturing, pre-clinical and clinical information for the prototype candidate to be moved forward in development. Each subsequent child IND submitted by the same sponsor/investigator would crossreference those sections of the "parent" IND that were in common, while providing additional unique "child"-specific information.

An exploratory parent-child IND is a feasible approach to reducing the regulatory procedural burden associated with evaluating multiple highly related T-cell-based therapeutic constructs or manufacturing alterations in small clinical studies. The parent IND would contain sections providing all of the common information relevant for the tobe-tested initial candidates or manufacturing alterations. For each candidate or manufacturing alteration, a child IND would also be submitted. This child IND would depend on heavy cross-referencing to the common sections in the parent IND while providing only the candidate- or process-specific information (e.g., chemistry, manufacturing, and controls [CMC] or non-clinical data) in separate sections. We note that cross-referencing, for example, by an academic investigator to previously submitted information in another IND, with appropriate authorization, is an accepted current practice.

At the time of initial IND submission, the parent and child IND could be assigned separate IND numbers, to facilitate safety reporting and so on, but reviewed in parallel within the standard 30-day IND review window. The exploratory IND would include an explicit agreement by the sponsor that once the early testing of a particular construct or process is completed or discontinued, the associated exploratory child IND would be withdrawn. If the sponsor intends to proceed with full development of a candidate or manufacturing process, a new, traditional IND would be submitted for that candidate. Prior clinical experience with the candidate might result in an expedited review of the new product development IND. Subsequent candidates or processes consistent with the common information in the original parent IND could subsequently be added as additional children to the original parent, again relying heavily on cross-references.

Because the time and resource savings associated with the use of parent-child INDs would only be realized in situations where most of the information contained in the parent IND would be relevant to all of the investigational candidates, the use of parent-child INDs would be limited to situations where the commonalities between the early cellular therapy candidates or manufacturing interventions are great enough to produce real gains in efficiency for both the sponsor and the FDA reviewing division. For example, an exploratory IND might be limited to candidates directed at the same target, even if in multiple tumor types (e.g., neurotrophic tyrosine kinase receptor 1 expressing non-small cell lung cancer, or triple negative breast cancer). Whether a parent-child IND is appropriate for a particular set of candidates could be discussed in an INTERACT (Initial Targeted Engagement for Regulatory Advice on Center for Biologics Evaluation and Research products) or pre-IND meeting or the justification could be provided in the IND itself (with an associated risk of delay if the FDA disagrees). Given the potential efficiencies gained, the parent-child IND concept is likely to be embraced by academic- and industry-sponsored researchers moving forward and warrants additional discussions with the FDA in the context of specific development programs.

\section{Pathways to Enable Manufacturing and Testing Evolution During Late-stage Development and Post Licensure}

In the case of T-cell-based therapeutics and other cell-based therapies, making these products effective for the greatest number of patients may require adjusting manufacturing parameters for specific patient subsets. The single manufacturing process framework is often chosen for regulatory expediency and a lack of product knowledge to discriminate between patients. At the same time, patient-to-patient variability in the quality of T cells from these patients can lead to suboptimal drug product quality for a subset of patients when a single manufacturing process is used for all patients. It may be more appropriate to adapt the manufacturing process for a subset of patients to increase the efficacy for the specific patient cohort, without impacting safety and efficacy for patients already responding using the original manufacturing process.

These new process parameter combinations for patient subsets are discovered during clinical development as more patients are treated and more clinical, translational and product quality data are collected. As product and process knowledge increases, a regulatory strategy that adjusts a process based on patient or patient-specific raw material information to maximize product quality for all patients will be necessary without conducting extensive costly and lengthy clinical studies. Current regulatory requirements and processes may not readily allow for patient-level modifications, especially when the understanding of the linkage among product quality attributes, manufacturing processes, clinical efficacy and safety continue to evolve late in development or after licensure.

Traditionally, manufacturing processes are locked in advance of late-stage clinical trials to be able to repeatedly measure effect across many patients. As product and process knowledge becomes more comprehensive for cell therapies, it can be anticipated that an adaptive manufacturing process could be developed with the goal of 
generating highly similar drug product from variable patient-specific starting materials. The product and process knowledge to enable adaptive manufacturing in most cases will not emerge until a large number of patients are treated because the correlative analysis to discover the relationship is not available until the enrollment of the pivotal trial is already well advanced. An example of this type of relationship includes the frequency of specific T-cell subtypes [15].

\section{Using Post-Approval Lifecycle Management-like plan for making manufacturing and testing changes}

As we gain stronger product knowledge and process understanding and are able to correlate their impacts to clinical safety, efficacy and durability results, the insights gained are likely to lead to improvements that can be made to the manufacturing process and/or quality control tests, as anticipated in International Conference on Harmonisation of Technical Requirements for Registration of Pharmaceuticals for Human Use Q12 [16]. For example, based on data gained during clinical development, a process adaptation (e.g., culture medium optimization, culture condition optimization, analytics method improvement) is identified, which modestly improves manufacturing consistency, increases the efficacy or reduces adverse events (i.e., does not impact labeled dose). The magnitude of change in clinical benefit may not be large enough to justify a full clinical development but is still beneficial to patients. For these changes, modifications could be managed via a pre-negotiated plan with health authorities (e.g., Post-Approval Lifecycle Management [PALM] or Comparability Protocol). The filing requirements for the change may include a combination of an analytical comparability assessment, and/or a small clinical study, analogous to a bioequivalence study for a new process. A post-market commitment could be considered to demonstrate/confirm the efficacy of the new process.

\section{Create CMC commercial process change reporting categories for cell- based therapies}

The FDA December 2017 draft guidance for CMC changes to an approved application intended to assist manufacturers of biological products in assessing the reporting category for $\mathrm{CMC}$ changes. This guidance provides a starting framework that can be further extended to T-cell-based therapies. As the cell-based therapeutic industry accumulates commercial manufacturing experience, sponsors can identify the most frequent manufacturing changes and propose recommended reporting categories based on risk assessment: Annual Reportable (AR), Changes Being Effected-0, Changes Being Effected-30 or PostApproval Supplement. Consistent with the fundamental guiding principle from the biologics guideline, the reporting category selected should be commensurate with the risk of an unintended outcome resulting from changes involving these elements. When assessing the impact of change on product quality, the historical product and process knowledge including experience gained during commercial manufacturing should be fully leveraged. Developing a best practice guide for cell therapy with specific examples of process and testing changes for the range of categorization would be a beneficial activity, which could be created by an industrial consortium.

However, it should be noted that the overall variability in cell-based therapy processes is influenced by the incoming patient-to-patient variability. Therefore, the traditional process performance qualification approach using three healthy donor batches to qualify each change has limited applicability and, instead, a rigorous, continuous process verification (CPV) plays a larger role in demonstrating process control. Use of healthy donors to characterize process and analytical variability in theory is a good approach, but a significant number of healthy donors are potentially needed to quantify the variability contribution of the process and analytics. This consumes resources and manufacturing capacity that otherwise would be used to produce clinical or commercial products. Hence, a concurrent qualification approach, where a change is introduced in manufacturing based on small-scale data and is subject to verification through a CPV program during clinical/commercial use, is not only more efficient but would also allow the confirmation of change in the setting of real patients instead of healthy donors. In addition, stand-alone qualification of the specific process or manufacturing change on a risk-based impact assessment without the need for endto-end full process performance qualification may be sufficient in some cases (e.g., a change in a supplier of raw materials, reagents and solvents that have a minimal potential to affect product quality) provided that the materials' specific use, physicochemical properties, impurity content and acceptance criteria remain comparable could be validated offline and reported as an AR. Additionally, a change from a manual operation to an automated operation that does not change the process parameter set points could be addressed through automation qualification and reported as an AR.

Lastly, in some cases, demonstrating analytical comparability at the appropriate in-process intermediate level may be sufficient. For example, demonstration of comparability for the vector bulk material due to a process change in the vector manufacturing process should not require demonstration of final product comparability post-transduction. Analytical comparability of the bulk viral vector and, if needed, use of a small-scale model to confirm transducibility of the cellular inprocess product at the same transduction levels should be considered sufficient. The life cycle plan for process and method changes needs to be carefully sequenced so that potential impact of the changes is seen throughout the CPV program. Changes to process parameters outside of previously validated ranges should be assessed with respect to criticality to process performance and product quality.

The reporting categories and extent of requalification for these changes will be assessed keeping the above considerations in mind. A risk-based approach to determine the extent and approach of qualification should be used that would determine if (i) qualification can be performed using small-scale or whether full-scale confirmation is needed; (ii) qualification exercise can be limited to evaluating product attributes of the impacted intermediate or the final drug product; and (iii) separate qualification is needed or if heightened CPV program for a period of time can be used. Given that many cell therapy companies are focused on early access to the promising therapies, several process improvements are deferred and become part of the post-approval life cycle plan (Box 1).

Box 1. Examples of such deferred changes.

1) New primary packaging components for the final product to simplify ease of administration and enable more clinical sites

2) New activation reagents; introduction of a new media processing system to improve manufacturing robustness

3) A higher-grade of fetal bovine serum to improve reliability

4) Change of buffer manufacturer from in-house to an external manufacturer

5) Automation of manual processing steps

6) Automation of flow cytometry data analysis

7) Increase in vector production scale to meet increasing demand

8) Change to a rapid sterility method

9) Rapid microbiological testing

10) Change of vector manufacturing process to a suspension cell culture process

11) The addition of an identical manufacturing suite to double capacity for both vector and drug product

12) Change in the antibiotic resistance in the vector cell bank/ plasmid; improved potency method

13) Change to stability data for expiry extension 
Quality standards for ancillary materials used in the manufacturing of cell-based therapy products intended to be developed as commercial products

Currently, sponsor companies are restrained by the limited numbers of GMP producers of these ancillary materials (e.g., recombinant proteins, growth factors, cytokines and small molecules) because of the regulatory requirements associated with choosing novel reagents. For the foreseeable future, the supply chain will be a critical path for product commercialization. The root cause for this limited supply chain is multi-factorial, but some modifications of applicable regulatory guidance could accelerate innovation.

Stakeholders desire more uniform feedback from within health authorities around quality and testing standards for non-GMP ancillary materials. Stronger guidance on how to stratify quality and/or characterization requirements based on whether they are excipients, product contacting (primary) or secondary ancillary material (e.g., plasmids used in viral vector manufacturing) or tertiary ancillary materials would be beneficial to the field. Moreover, greater health authority alignment with the principles published in United States Pharmacopeia $<1043>$ or other guidance documents could result in greater consistency in CMC development across multiple phases.

\section{Conclusion}

The "Designing the Future of Cellular Therapies" meeting brought together a group of stakeholders from academia, biotechnology, pharmaceuticals, government, regulatory agencies, patient advocates and non-profits. Several opportunities and strategies that could expedite T-cell-based therapies into first-in-human studies and ensure that T-cell-based therapeutics are impactful for the greatest number of patients were discussed (Table 2). Ongoing dialogue and collaboration with the FDA and other global health authorities to increase the clarity and uniformity of regulatory requirements will be critical to the development of the field. Moreover, efforts to encourage transparency, collaboration and data sharing are needed so changes can be appropriately monitored, which would allow the field to adapt to improvements efficiently. The proposals could be particularly useful in bringing cutting edge biological and genetic approaches forward to enhance the potential of the next generation of cell therapies to demonstrate efficacy in the highly complicated tumor microenvironment and extend these promising new therapies to the many patients with life-threatening solid tumors with no remaining treatment options.

\section{Funding}

This work was supported by the Friends of Cancer Research and the Parker Institute for Cancer Immunotherapy.

\section{Declaration of Competing Interest}

Jeff Bluestone is employed by Sonoma BioTherapeutics, a Treg cell therapy company, and reports grants and personal fees from Juno, nonfinancial support from Becton Dickenson, and has several issued and pending patents. Lisa Butterfield received consulting honoraria from Calidi Biotherapeutics, SapVax, NextCure, Replimmune, Western Oncolytics, Torque Therapeutics and Phyxis Oncology and has stock options in Calidi Biotherapeutics, NextCure and Phyxis Oncology. Ramy Ibrahim is an observer on the board of directors for Lyell Immunopharma and serves on the Scientific Advisory Board for Bit Bio. Michael Kalos is an employee of Arsenal Biosciences and has stock in Arsenal Biosciences and Johnson and Johnson. Anne Keane is employed by Lyell Immunopharma. Chin Koerner is employed by Novartis. Ann Lee is employed by Juno Therapeutics, A Bristol Myers Squibb Company. Bruce Levine is on the Scientific Advisory Board for Avectas, Brammer Bio, Incysus, CRC Oncology/ Cure Genetics, Vycellix and Ori Biotech, received consultancy fees from Brammer Bio, Incysus and CRC Oncology/Cure Genetics, has patents and royalties with and received research funding from Novartis Pharmaceuticals Corporation and holds equity ownership in and received research funding from Tmunity Therapeutics. Ingrid Markovic is employed by Genentech. Tim Moore is employed by PACT Pharma. Chris Ramsborg is employed by Juno Therapeutics, A Bristol Myers Squibb Company. Bruce Thompson is an employee of Lyell Immunopharma. Yuan $\mathrm{Xu}$ is employed by Legend Biotech and currently owns equity in Merck.

\section{Author Contributions}

Conception and design of the study: MDS, AK, LHB, BLL, RI, JB, ES, JA. Acquisition of data: MDS, AK LHB, BLL, BT YX, CR, AL, MK, CK, TM, IM. Analysis and interpretation of data: MDS, AK, LHB, BLL, BT, YX, CR, AL, MK, CK, TM, IM, LL, RI, JB, ES, JA. Drafting or revising the manuscript: MDS, AK, LHB, BLL, BT, YX, CR, AL, MK, CK, TM, IM, LL, RI, JB, ES, JA. All authors have approved the final article.

\section{Acknowledgements}

The authors also thank representatives of the FDA for their review of the manuscript. This manuscript was developed based on working group discussions and public discussions at the meeting titled "Designing the Future of Cell Therapies" as well as a roundtable discussion in Washington, DC, supported by Friends of Cancer Research and Parker Institute for Cancer Immunotherapy (meeting agenda and recap: https://www.focr.org/events/future-cell-therapies). Attendees of the roundtable are provided in Supplemental Table 2. The authors would like to thank the meeting participants and the panelists who facilitated the discussions and whose views contributed to the information provided in this article.

\section{Supplementary materials}

Supplementary material associated with this article can be found in the online version at doi:10.1016/j.jcyt.2020.01.014.

\section{References}

[1] Stevanović S, et al. Landscape of immunogenic tumor antigens in successful immunotherapy of virally induced epithelial cancer. Science 2017;356(80):200LP-205.

[2] Zacharakis N, et al. Immune recognition of somatic mutations leading to complete durable regression in metastatic breast cancer. Nat. Med. 2018;24:724-30.

[3] Tran E, et al. Cancer Immunotherapy Based on Mutation-Specific CD4+ T Cells in a Patient with Epithelial Cancer. Science 2014;344(80):641LP-645.

[4] Angelo SP, et al. Antitumor Activity Associated with Prolonged Persistence of Adoptively Transferred NY-ESO-1 c259 T Cells in Synovial Sarcoma. Cancer Discov. 2018:8:944LP-957.

[5] Brown CE, et al. Regression of Glioblastoma after Chimeric Antigen Receptor T-Cell Therapy. N. Engl. J. Med. 2016;375:2561-9.

[6] NCI SEER. Cancer Stat Facts: Cancer of Any Site. Available at: https://seer.cancer. gov/statfacts/html/all.html. (Accessed: 14th August 2019)

[7] FDA. Guidance for Industry, Investigators, and Reviewers: Exploratory IND Studies. (2006). Available at: https://www.fda.gov/media/72325/download. Accessed: 14th August 2019.

[8] FDA. Master Protocols: Efficient Clinical Trial Design Strategies To Expedite Development of Oncology Drugs and Biologics. (2018). Available at: https://www.fda. gov/media/120721/download. Accessed: 14th August 2019.

[9] FDA. Adaptive Design Clinical Trials for Drugs and Biologics. (2018). Available at: https://www.fda.gov/media/78495/download. Accessed: 14th August 2019.

[10] FDA. Guidance for Industry: CGMP for Phase 1 Investigational Drugs. (2008). Available at: https://www.fda.gov/media/70975/download. (Accessed: 14th August 2019) 
[11] Friends of Cancer Research \& Parker Institute for Cancer Immunotherapy. Designing the Future of Cell Therapies. (2019). Available at: https://www.focr.org/sites/ default/files/pdf/Friends_Cellular_Therapies_White_Paper.pdf. Accessed: 14th August 2019.

[12] Cornetta K, et al. Absence of Replication-Competent Lentivirus in the Clinic: Analysis of Infused T Cell Products. Mol. Ther 2018:26:280-8.

[13] Cornetta K, et al. Screening Clinical Cell Products for Replication Competent Retrovirus: The National Gene Vector Biorepository Experience. Mol. Ther. - Methods Clin. Dev. 2018;10:371-8.
[14] Skrdlant LM, Armstrong RJ, Keidaisch BM, Lorente MF, DiGiusto DL. Detection of Replication Competent Lentivirus Using a qPCR Assay for VSV-G. Mol. Ther. Methods Clin. Dev. 2017;8:1-7.

[15] Fraietta JA, et al. Determinants of response and resistance to CD19 chimeric antigen receptor (CAR) T cell therapy of chronic lymphocytic leukemia. Nat. Med. 2018;24:563-71.

[16] FDA. Q12 Technical and Regulatory Considerations for Pharmaceutical Product Lifecycle Management Core Guideline Guidance for Industry. (2018). Available at: https://www.fda.gov/media/113483/download. Accessed: 14th August 2019. 